\title{
LncRNA HOXC-AS3 Promotes Epithelial-to- mesenchymal Transition through Decreasing the Expression of FBXL17 in Cervical Squamous Cell Carcinoma
}

\section{Chengyin Weng}

Guangzhou First People's Hospital

\section{Xia Liu}

Guangzhou First People's Hospital

Haibo Mao

Guangzhou First People's Hospital

\section{Xisheng Fang}

Guangzhou First People's Hospital

\section{Baoxiu Li}

Guangzhou First People's Hospital

\section{Lina Wang}

Guangzhou First People's Hospital

\section{Guolong Liu}

Guangzhou First People's Hospital

Lin Lv ( $\nabla$ eylinlv@scut.edu.cn )

Guangzhou First People's Hospital

\section{Research Article}

Keywords: Long noncoding RNA HOXC-AS3, oncogenic IncRNA, Cervical squamous cell carcinoma, FBXL17, EMT

Posted Date: June 10th, 2021

DOl: https://doi.org/10.21203/rs.3.rs-598314/v1

License: (1) This work is licensed under a Creative Commons Attribution 4.0 International License. Read Full License 


\section{Abstract}

\section{Background:}

The expression and biological roles of a novel IncRNA HOXC-AS3 in cervical squamous cell carcinoma (CESC) are largely unknown.

\section{Methods:}

LncRNA HOXC-AS3 was identified from The Cancer Genome Atlas (TCGA) database. Real time quantitative PCR (qRT-PCR) was used to evaluate the expression of HOXC-AS3 in 68 pairs of CESC tumor tissues and adjacent normal tissues. Kaplan-Meier survival analysis was used to assess the significance of HOXC-AS3 in predicting overall survival (OS). Correlations between HOXC-AS3 expression and clinicopathological parameters were assessed using Chi-square test. The biological functions of HOXCAS3 in CESC were investigated using loss-of and gain-of function assays, which including cell proliferation, migration and invasion. Furthermore, the underlying mechanism was revealed using bioinformatics prediction, qRT-PCR and western blot.

\section{Results:}

HOXC-AS3 expression was significantly up-regulated in CESC tumor tissues. Up-regulated HOXC-AS3 expression was correlated with advanced stage and poor OS. Over-expression of HOXC-AS3 promoted tumor cell proliferation, migration and invasion. Conversely, HOXC-AS3 knockdown significantly inhibited these effects. Subcellular fractionation analysis revealed that HOXC-AS3 was mainly localized in cell nuclei. Further mechanistic analyses demonstrated that HOXC-AS3 down-regulated the expression of FBXL17. The promotive effects of HOXC-AS3 on cell proliferation, migration and invasion could be repressed by FBXL17. Moreover, HOXC-AS3 activated the EMT process to promote the progression of CESC.

\section{Conclusion:}

In conclusion, our study indicated that HOXC-AS3 acted as an oncogenic IncRNA in CESC through downregulating FBXL17 expression and activating EMT process.

\section{Background}

Cervical squamous cell carcinoma (CESC) is the most common type of cervical cancer and represents the third most common cancers and the fourth leading cause of cancer-related death in women worldwide [1, 2]. There are 530,000 newly diagnosed cases and 270,000 deaths annually [1]. Most of the patients were diagnosed at advanced stage, thus the treatment strategies are limited, especially for the patients with distant metastasis [3-5]. Therefore, further study is needed to explore new biomarkers which could be used in the diagnosis and treatment of CESC patients. 
Long non-coding RNAs emerge as oncogenes or tumor suppressors in various malignancies [6].

Dysregulated expression of IncRNAs are involved in multiple cellular process, including cell proliferation, migration and invasion in CESC [7, 8]. In addition, IncRNAs regulated the expression and functions of targeted genes at various levels, including chromosomal, transcriptional and post-transcriptional levels [9-11]. For example, IncRNA SNHG12 was significantly upregulated in CESC and overexpression of SNHG12 promoted cell migration, invasion and epithelial-mesenchymal transition in CESC [12]. Feng et al found that IncRNA-CTS promoted the cell migration, invasion and metastasis of cervical cancer through promoting EMT process [13]. LncRNA 799 promoted the metastasis of cervical cancer through upregulating the expression of TBL1XR1 [14].

In this study, we identified differentially expressed IncRNA HOXC-AS3 between CESC tumor tissues and normal tissues from the TCGA database. HOXC-AS3, as an antisense transcript partner of HOXC10, was reported to play important roles in some malignant tumors, including gastric cancer and lung cancer [15, 16]. Upregulated expression of HOXC-AS3 was identified in gastric cancer tissues and correlated with poor overall survival of gastric cancer patients [15]. In addition, HOXC-AS3 promoted invasive mucinous adenocarcinoma progression by modulating FUS/FOXM1 expression [16]. However, the expression and biological functions of HOXC-AS3 in CESC are largely known.

In this study, the expression of HOXC-AS3 was compared between CESC tumor tissues and adjacent normal tissues. The associations between HOXC-AS3 expression and clinical parameters and overall survival were assessed. Moreover, the functional experiments were performed to investigate the roles of HOXC-AS3 in cell proliferation, migration and invasion. Subsequently, the potential mechanism of HOXCAS3 in promoting the progression of CESC was further revealed. Our results demonstrated that HOXC-AS3 contributed the malignant behaviors of CESC, thus offering novel diagnosis and therapeutical target for CESC patients.

\section{Results}

\section{Expression and clinical significance of HOXC-AS3 in cervical squamous cell carcinoma (CESC)}

The differentially expressed IncRNAs were identified from TCGA dataset. There were 255 cases of CESC tissues and 2 normal tissues. The expression of HOXC-AS3 was significantly up-regulated in tumor tissues based on the TCGA database (Fig. 1A) and GEPIA (Fig. 1B). These results were verified by comparing the expression of HOXC-AS3 in 68 pairs of CESC tumor tissues and adjacent normal tissues using real time quantitative PCR (qRT-PCR) $(P<0.01$, Fig. 1C).

The associations between HOXC-AS3 expression and various clinic-pathological parameters were assessed and demonstrated in Table 1. Higher HOXC-AS3 expression was positively correlated with advanced FIGO stage $(P=0.033)$ and lymph node metastasis $(P=0.049)$, but not with age, gender, tumor 
size and differentiation status. Up-regulated expression of HOXC-AS3 was positively correlated with poor overall survival of CESC patients (Fig. 1C, P).

Table 1

The correlations between HOXC-AS3 expression and

clinicopathological parameters of cervical squamous cell carcinoma

(CESC) patients

\begin{tabular}{|c|c|c|c|c|}
\hline \multirow[t]{2}{*}{ Clinical parameters } & \multicolumn{2}{|c|}{ HOXC-AS3 } & \multirow[t]{2}{*}{ Chi-square } & \multirow[t]{2}{*}{$P$-value } \\
\hline & Low & High & & \\
\hline \multicolumn{5}{|l|}{ Age } \\
\hline$\geqq 50$ & 16 & 22 & 2.147 & 0.143 \\
\hline$<50$ & 18 & 12 & & \\
\hline \multicolumn{5}{|l|}{ Tumor size (cm) } \\
\hline$<4$ & 10 & 5 & 0.963 & 0.326 \\
\hline$\geqq 4$ & 24 & 22 & & \\
\hline FIGO stage & & & 4.533 & $0.033^{*}$ \\
\hline I/II & 28 & 20 & & \\
\hline III & 6 & 14 & & \\
\hline Lympho node metastasis & & & 3.886 & $0.049^{*}$ \\
\hline Positive & 18 & 10 & & \\
\hline Negative & 16 & 24 & & \\
\hline Differentiation & & & 0.003 & 0.953 \\
\hline Well to Moderate & 23 & 18 & & \\
\hline Poor & 21 & 16 & & \\
\hline
\end{tabular}

Moreover, Kaplan-Meier survival analysis demonstrated that CESC patients with high HOXC-AS3 expression had worse OS than patients with low HOXC-AS3 expression ( $P=1.572 \mathrm{e}-02$, Fig. 1D).

The HOXC-AS3 expression in normal cervical epithelial cell line HacaT and different CESC cell lines (SiHa, Hela and C33A) were compared using qRT-PCR. As show in Fig. 1E, the expression of HOXC-AS3 was much higher in CESC tumor cells than normal cell line. Taken together, these data indicated that HOXCAS3 acted as an oncogenic biomarker in CESC. 


\section{HOXC-AS3 promotes cell proliferation, migration and invasion}

The expression of HOXC-AS3 in various CESC cell lines were compared using qRT-PCR (Fig. 1E). SiHa demonstrated relative high HOXC-AS3 expression, while C33A demonstrated with relative low HOXC-AS3 expression. Thus, we constructed HOXC-AS3 overexpression cells (oe-HOXC-AS3) by transfecting HOXCAS3 expressing plasmid (pcDNA3.1-HOXC-AS3) or empty vector control to cell line. HOXC-AS3 knockdown cell line was constructed by transfecting cells with short hairpin RNA (shRNAs). The expression of HOXC-AS3 in different groups were analyzed using qRT-PCR (Fig. 2A). The effect of HOXCAS3 on cell proliferation was assessed using CCK8 assay. As shown in Fig. 2, over-expression of HOXCAS3 promoted tumor cell proliferation (Fig. 2B, $\mathrm{P}<0.001$ ), while knockdown of HOXC-AS3 significantly repressed cell proliferation (Fig. 2 C, $\mathrm{P}<0.001$ ).

The cell migration and invasion were analyzed using transwell assays. HOXC-AS3 over-expression promoted the migration and invasion of CESC cells (Fig. 2D and E). Figure 2D showed the representative microscopic images. Conversely, knockdown of HOXC-AS3 resulted in significant inhibition on cell migration and invasion (Fig. 2F and G). Taken together, these data suggested that HOXC-AS3 promoted CESC cell proliferation, migration and invasion.

\section{Down-regulation of FBXL17 expression by HOXC-AS3}

We further investigated the mechanisms by which HOXC-AS3 promoted CESC cell proliferation, migration and invasion. Subcelluar fractionation assay showed that HOXC-AS3 was mainly localized in the nuclei of CESC cells (Fig. $3 \mathrm{~A}$ ), which indicating that HOXC-AS3 might directly interact with target mRNAs. Potential mRNAs that could interact with HOXC-AS3 were predicted using R software. And FBXL17 was identified as a potential target mRNA for HOXC-AS3. There was negative correlation between HOXC-AS3 expression and FBXL17 expression (Fig. 3B). Over-expression of HOXC-AS3 down-regulated the expression of FBXL17 (Fig. 3C), while knockdown of HOXC-AS3 up-regulated the expression of FBXL17 (Fig. 3D).

Moreover, the expression of FBXL17 were assessed through GEPIA online database and our clinical samples. As shown in Fig. 3E, the expression of FBXL17 was significantly down-regulated in CESC tumor tissues than normal tissues according to the TCGA database. Analyzing the 68 paired tissue specimens, we found down-regulated expression of FBXL17 in CESC tumor tissues (Fig. 3F). Pearson correlation coefficient also verified that there was inverse correlation between HOXC-AS3 expression and FBXL17 expression according to our qRT-PCR results (Fig. 3G). These data suggested that IncRNA HOXC-AS3 repressed the expression of FBXL17. 


\section{HOXC-AS3 promoted CESC cells proliferation, migration and invasion trough down-regulating the expression of FBXL17}

We next investigated whether the effect of HOXC-AS3 on cell proliferation, migration, invasion and EMT process by regulating FBXL17. HOXC-AS3 overexpression cells were transfected with oe-FBXL17 plasmid. As shown in Fig. 4A, the promotive effect of HOXC-AS3 on cell proliferation was repressed by FBXL17 overexpression. In addition, concomitant overexpression of FBXL17 and HOXC-AS3 significantly decreased the migration and invasion abilities induced by HOXC-AS3 overexpression (Fig. 4B and C). These data indicated that HOXC-AS3 promotes the proliferation, migration and invasion of CESC cells through down-regulating the expression of FBXL17.

\section{HOXC-AS3 promotes the EMT process of CESC cells}

We further studied whether EMT contributed to HOXC-AS3-induced migration and invasion of CESC cells. Firstly, the mRNA expression levels of EMT makers E-cadherin, N-cadherin and Vimentin were detected using QRT-PCR. Overexpression of HOXC-AS3 down-regulated the expression of E-cadherin (Fig. 5A), and up-regulated the expression of N-cadherin (Fig. 5B) and Vimentin (Fig. 5C). Overexpression of FBXL17 reversed the EMT promotive role of HOXC-AS3 overexpression. Similarly, the expression of EMT makers at protein level were analyzed by western blot. As shown in Fig. 5D, overexpression of HOXC-AS3 promoted the EMT process of CESC cells by upregulating E-cadherin expression and downregulating $\mathrm{N}$ cadherin and Vimentin expression. Overexpression of FBXL17 counteracted the promotive role of HOXCAS3 in the EMT process. Taken together, these data revealed that HOXC-AS3 promoted the EMT process of CESC cells, which depending on the decreased expression of FBXL17.

\section{Discussion}

Long noncoding RNAs emerges as critical regulators in cancer development. LncRNAs could regulate gene expression and participate in multiple molecular biological processes through various mechanisms, such as miRNA sponge, RNAi, gene co-suppressing, et al [17]. In addition, IncRNAs could recruit RNA binding proteins to stabilize target gene mRNA [18-20]. HOXC-AS3 was reported to be upregulated in some solid tumors, including gastric cancer and invasive mucinous adenocarcinoma. However, little is known about the expression and biological functions of HOXC-AS3 in cervical cancer.

In this study, we firstly investigated the expression and prognostic value of IncRNA HOXC-AS3 in cervical squamous cell carcinoma. HOXC-AS3 expression was significantly upregulated in CESC tumor tissues compared with normal tissues. High expression of HOXC-AS3 was positively correlated with poor overall survival. Accordingly, HOXC-AS3 was highly expressed in multiple cancers, including glioblastoma, gastric cancer and invasive mucinous adenocarcinoma of the lung [15]. 
All these studies indicated that HOXC-AS3 worked as an oncogenic IncRNA in solid tumors.

Moreover, the potential biological roles and underlying mechanisms of HOXC-AS3 in CESC are largely unknown. In vitro studies demonstrated that overexpression of HOXC-AS3 promoted CESC cell proliferation, migration and invasion. Conversely, silence of HOXC-AS3 inhibited these effects. Thus, our study indicated that HOXC-AS3 could act as an oncogene and promote the progression of CESC. Subcellular fractionation assay showed that HOXC-AS3 was mainly localized in the nuclei of CESC cell, which indicating that HOXC-AS3 might directly interact with target genes to regulate the biological functions of CESC.

Zhang et al found that the gain of H3K4me3 and H3K27acetylation could activate HOXC-AS3 expression in bot $\mathrm{h}$ gastric cancer tissues and cell lines [15]. HOXC-AS3 could promote the proliferation and migration of gastric cancer cells by directly interacting with YBX1 [15]. HOXC-AS3 bound to HOXB13 and promoted the proliferation, migration and invasion of glioblastoma cells [21]. HOXC-AS3 promoted the osteogenesis of multiple myeloma derived mesenchymal stromal cells [22]. Yang et al. demonstrated that HOXC-AS3 promoted the progression of invasive mucinous adenocarcinoma of lung by recruiting FUS to stabilize FOXM1 [6]. Shi et al revealed that HOXC-AS3 promoted breast cancer metastasis by sponging miR-3922-5p to upregulated the expression of PPP1R1A [23]. Here, we identified FBXL17 as the downstream target of HOXC-AS3. HOXC-AS3 interacted with FBXL17 and negatively regulated its expression. Here, we found down-regulated expression of FBXL17 in CESC tumor tissues. Similarly, Mason et al found that FBXL17 worked as a tumor suppressor in breast cancer by decreasing global $O$ GIcNAcylation[24]. FBXL17 ubiquitylation of Sufu repressed medulloblastoma cell proliferation by regulating Hedgehog signaling pathway [25]. Moreover, cellular biological function assays showed that overexpression of FBXL17 could counteract the promotive effects of HOXC-AS3 on cell proliferation, migration and invasion, which also suggesting that the oncogenic function of HOXC-AS3 relied on the FBXL17. Finally, we found that HOXC-AS3 interacted with FBXL17 to regulate the EMT process to promote the progression of CESC.

\section{Conclusion}

In conclusion, we found a novel oncogenic IncRNA HOXC-AS3 in CESC. Further study revealed that HOXCAS3 promoted the EMT process by down-regulating the expression of FBXL17. This study identified a novel biomarker which could predict the survival and potential metastatic capability in CESC.

\section{Material And Methods}

\section{Ethical statement}

There were 68 cases of cervical squamous cell carcinoma patients included in this study. All the patients were treated with surgery between January 2015 and December 2016. None of the patients were treated 
with any prior anticancer therapies and were histopathologically confirmed as cervical squamous cell carcinoma. Ethics Committee of Guangzhou First People's Hospital approved this study. All the patients were informed and consent was obtained by all the patients.

\section{Experiments statement}

All methods were carried out in accordance with relevant guidelines and regulations.

\section{RNA-seq data of CESC}

RNA-seq data of CESC was downloaded from the TCGA database. The data was retrieved from the TCGA using perl software and analyzed using R software.

\section{Cell line and cell culture}

The human CESC cell lines (SiHa, Hela and C33A) and human keratinocytes cell line HacaT cells were purchased from the Cell Bank of the Chinese Academy of Science (Shanghai, China) and were cultured in RMPI1640 or DMEM supplemented with $10 \%$ fetal bovine serum (FBS).

\section{Cell transfection}

The short hairpin RNA (shRNA) targeting HOXC-AS3 and scrambled negative controls were purchased from GenePharma (Shanghai, China). The cell transfection was conducted using Lipofectamine 2000 Transfection Reagent (Invitrogen) for $24 \mathrm{~h}$ according to the manufacturer's instructions.

\section{Lentivirus infection}

To construct an overexpression plasmid, cDNA sequence of HOXC-AS3 or FBXL17 was subcloned into the pcDNA3.1 expression vector by GeneCoppeia (Guangzhou, China). C33A cells were infected with lentivirus in the presence of polybrene and selected with puromycin to establish stale cells

\section{Nucleus and cytoplasm fraction isolation}

Cell cytoplasmic and nuclear fractions were isolated using Nuclear/Cytosol Fraction Kit (Biovision, CA, USA) following the manufacturer's instructions. Briefly, the cells were incubated with cytosolic extraction buffer for 15 mins. After centrifugation, the cytosol supernatants were harvested and the following nuclear fractions were incubated with nuclear extraction buffer. After centrifugation, the nuclear fraction 


\section{Real time quantitative PCR (qRT-PCR)}

Total RNA from tissues and cell lines were extracted using TRIzol reagent (Invitrogen, USA) according to the manufacturer's instructions. The concentration of total RNA was assessed using NanoDrop 2000 (Thermo). The cDNA was synthesized using Takara cDNA Synthesis Kit. The resulting cDNA was performed to qRT-PCR on an Applied Biosystems 7900HT Fast Real-Time. The relative mRNA levels of genes were calculated based on the Ct value and normalized to the internal control GAPDH or U6. The primers used for qRT-PCR were as follows: 5'-CGTGCGCTCTGTAAAGG-3' (F) and

5'-AGCCTCGGTCAGTTTGG-3' (R) for HOXC-AS3;

5'-ACTGGCGTGACCTTTGTTTAG-3' (F) and 5'-CAGACATACTGCGACAATCAGAA-3' (R) for FBXL17;

5'-AAACAGTAGTGGAGAATCAAGCC-3' (F) and

5'-AGAACTCGAACCAGATCCAGAG-3' (R) for E-cadherin; 5'-AGTCAGTCGGAAAGTGAGCAG-3' (F) and

N-cad-R 5'-ACATCAGCTATCCGTTCCTTCT-3' (R) for N-cadherin;

5'-TGTGCCTCAGATCAACTGCG-3' (F) and

5'-GCATACACGTCAGTGCCATAAA-3' (R) for Vimentin; 5'-CTCCTCCTGTTCGACAGTCAGC-3' (F) and 5'CCCAATACGACCAAATCCGTT-3' (R) for GAPDH; 5'-TTATGGGTCCTAGCCTGAC-3' (F) and 5'CACTATTGCGGGCTGC-3' (R) for U6.

\section{Western blot}

Total protein was extracted from tissues and cell lines using RIPA solution as previously described. The resulting protein lysates were separated by $10 \%$ sodium dodecyl sulfate poly-acrylamide gel electrophoresis (SDS-PAGE). After being transfected to a PVDF membrane and blocked, the membrane was incubated with the first antibodies at $4^{\circ} \mathrm{C}$ overnight, which including Anti-E-cadherin (AF0131), anti$\mathrm{N}$-cadherin (AF4039) and anti-Vimentin (AF7013). All the antibodies were purchased from Affinity Biosciences. After washing, the membrane was incubated with secondary antibodies. Then the results were developed using an enhanced chemiluminescence detection system.

\section{Cell proliferation}


The cell proliferation was assessed using Cell Counting Kit-8 assay. Indicated cells were seeded in a 96well plate at a concentration of 5,000 cells per well and collected at different time point, including 0,24 , $48,72 \mathrm{~h}$. After being incubated with CCK8 reagent, the absorbance was measured using a microplate reader at a wavelength of $450 \mathrm{~nm}$.

\section{Transwell assay}

In vitro cell migration and invasion were investigated using a 24-well transwell inserts ( $8.0 \mu \mathrm{m}$, Corning). Briefly, for cell migration, $5 \times 10^{4}$ cells were suspended in $200 \mu \mathrm{l}$ serum free culture medium and placed in the upper chamber. For cell invasion, $5 \times 10^{4}$ cells were placed in the insert pre-coated with Matrigel. After incubation for $48 \mathrm{~h}$, the cells adhered to the lower surface of the membrane were stained with $0.1 \%$ crystal violet solution. The cells migrated or invaded in five randomly selected fields were calculated under a light microscope. Magnification, X100.

\section{Bioinformatics}

The potential targets of HOXC-AS3 were predicted using on a R software.

The correlation between HOXC-AS3 expression and FBXL17 expression was confirmed on an online tool GEPIA (http://gepia.cancer-pku.cn/).

\section{Statistical analysis}

The data were analyzed using GraphPad Prism 7 software. The comparison between two groups was calculated using student's $t$ test. The correlations between HOXC-AS3 expression and clinicopathological parameters were assessed using chi-square test. The correlation between HOXC-AS3 expression and overall survival was calculated using Kaplan-Meier survival curve with log-rank test. A two tailed $P$ value $<0.05$ was considered statistically significant.

\section{Abbreviations}

InRNA, long noncoding RNA; EMT, epithelial-to-mesenchymal transition; CESC, cervical squamous cell carcinoma; TCGA, The Cancer Genome Atlas; OS, overall survival; qRT-PCR, real time quantitative PCR.

\section{Declarations}

\section{Ethics approval and consent to participate}

There were 68 cases of cervical squamous cell carcinoma patients included in this study. Ethics Committee of Guangzhou First People's Hospital approved this study and all the patients were informed. 


\section{Consent for publication}

Not applicable

\section{Competing interests}

The authors declare that they have no competing interests

\section{Funding}

This research was funded by the Natural Science Foundation of Guangdong Province, China (No. 2021A1515011113), Guangzhou Science and Technology Program (No. 201904010427), and Guangzhou General Science and technology project of Health and Family Planning (grant number 20201A011007).

\section{Authors' contributions}

Chengyin Weng and Lin Lu conceived the project; Guolong Liu supervised this project; Chengyin Weng, Xia Liu and Lin Lu conducted the experiments and data analysis; Xiubao Li and Haibo Mao collected the clinical specimens; Xia Liu, Xisheng Fang, Lina Wang and Chengyin Weng collected clinical information; Lin Lu and Chengyin Weng drafted the manuscript; Guolong Liu revised the manuscript.

\section{Acknowledgements}

Not applicable.

\section{Availability of data and materials}

The datasets used and/or analysed during the current study are available from the corresponding author on reasonable request.

\section{References}

1. J F, I S, R D, S E, C M, M R, DM P, D F, F B: Cancer incidence and mortality worldwide: sources, methods and major patterns in GLOBOCAN 2012. International journal of cancer 2015, 136(5):E359386.

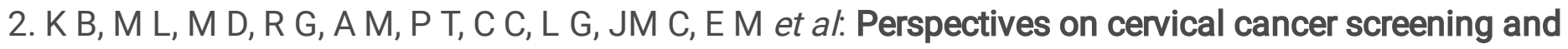
prevention: challenges faced by providers and patients along the Texas-Mexico border. Perspectives in public health 2019, 139(4):199-205. 
3. $L X, Y Y, M L, Y N Z, L Z, W L, X W, X X$ : CNN3 acts as a potential oncogene in cervical cancer by affecting RPLP1 mRNA expression. Scientific reports 2020, 10(1):2427.

4. N O, M W, S K, H M, H K, K K, T O, H T, T N, M S: Significance of concurrent use of weekly cisplatin in carbon-ion radiotherapy for locally advanced adenocarcinoma of the uterine cervix: A propensity score-matched analysis. Cancer medicine 2020, 9(4):1400-1408.

5. J Y, R D, J M, P M, J Y, C L, M R, T D, K B: Comparing survival outcomes between surgical and radiographic lymph node assessment in locally advanced cervical cancer: A propensity scorematched analysis. Gynecologic oncology 2020, 156(2):320-327.

6. J G, C D, XY, XB Y, F Z: Long noncoding RNA LINC00324 exerts protumorigenic effects on liver cancer stem cells by upregulating fas ligand via PU box binding protein. FASEB journal: official publication of the Federation of American Societies for Experimental Biology 2020.

7. J W, Z Z, H Q, C L, X C, Y Q, Q L: LncRNA NKILA inhibits the proliferation and promotes the apoptosis of CSCC cells by downregulating miRNA-21. Journal of cellular physiology 2020.

8. D X, P D, Y X, J Y, Y K, K I, N K, Y T, H W: MicroRNA-361-Mediated Inhibition of HSP90 Expression and EMT in Cervical Cancer Is Counteracted by Oncogenic IncRNA NEAT1. Cells 2020, 9(3).

9. $L Y Y, X Q, L L, J Z, M Z, X L, Y X, X J W, H X$ : The transcriptome profiles and methylation status revealed the potential cancer-related IncRNAs in patients with cervical cancer. Journal of cellular physiology 2019, 234(6):9756-9763.

10. L C, R K, C W, S W, Z L, S L, S L, T C, C M, S L: Circ-MALAT1 Functions as Both an mRNA Translation Brake and a microRNA Sponge to Promote Self-Renewal of Hepatocellular Cancer Stem Cells. Advanced science (Weinheim, Baden-Wurttemberg, Germany) 2020, 7(4):1900949.

11. H D, Y C: Competing endogenous RNA networks in cervical cancer: function, mechanism and perspective. Journal of drug targeting 2019, 27(7):709-723.

12. SY L, HM G, J L, LJ H, XL H, YH C, YH W, Y W, Q Y, JY Z: Long noncoding RNA SNHG12 modulated by human papillomavirus $16 \mathrm{E} 6 / \mathrm{E} 7$ promotes cervical cancer progression via ERK/Slug pathway. Journal of cellular physiology 2020.

13. S F, W L, X B, W P, Z J, S Z, Y Z, W T: LncRNA-CTS promotes metastasis and epithelial-tomesenchymal transition through regulating miR-505/ZEB2 axis in cervical cancer. Cancer letters 2019, 465:105-117.

14. LM L, FH Z, GJ Y, SF A, M Z, L H: Role of Long Noncoding RNA 799 in the Metastasis of Cervical Cancer through Upregulation of TBL1XR1 Expression. Molecular therapy Nucleic acids 2018, 13:580-589.

15. E Z, X H, C Z, J S, X L, X S, J C, D Y, L H, W D: A novel long noncoding RNA HOXC-AS3 mediates tumorigenesis of gastric cancer by binding to YBX1. Genome biology 2018, 19(1):154.

16. Z Y, T H: Long noncoding RNA HOXC-AS3 facilitates the progression of invasive mucinous adenocarcinomas of the lung via modulating FUS/FOXM1. In vitro cellular \& developmental biology Animal 2020, 56(1):15-23. 
17. C T, JH W, SKW T, T Z, TF C, TB N: LncRNAs with miRNAs in regulation of gastric, liver, and colorectal cancers: updates in recent years. Applied microbiology and biotechnology 2019, 103(12):46494677.

18. Z G, Z C, X Y, X H, N W, H W, C W, D G, F Z, M Y et al: Long noncoding RNA SchLAH suppresses metastasis of hepatocellular carcinoma through interacting with fused in sarcoma. Cancer science 2017, 108(4):653-662.

19. Y L, X L, M X, M L, M Y, M L, XM C, X L, R Z: The NF-kB-Responsive Long Noncoding RNA FIRRE Regulates Posttranscriptional Regulation of Inflammatory Gene Expression through Interacting with hnRNPU. Journal of immunology (Baltimore, Md: 1950) 2017, 199(10):3571-3582.

20. Y L, X Z, Q L, C L, R G-D, Z C, B S, JL F, J W, H H et al: IncRNA MIR100HG-derived miR-100 and miR$125 \mathrm{~b}$ mediate cetuximab resistance via Wnt/ $\beta$-catenin signaling. Nature medicine 2017 , 23(11):1331-1341.

21. X W, Y S, T X, K Q, B H, K Z, Z S, T Q, J S, L L: HOXB13 promotes proliferation, migration, and invasion of glioblastoma through transcriptional upregulation of IncRNA HOXC-AS3. Journal of cellular biochemistry 2019, 120(9):15527-15537.

22. B L, H H, S S, G F, HX, W Z, Y Q, C Q, Y W, Z Y et al: HOXC10 Regulates Osteogenesis of Mesenchymal Stromal Cells Through Interaction with Its Natural Antisense Transcript IncHOXC-AS3. Stem cells (Dayton, Ohio) 2019, 37(2):247-256.

23. SH S, J J, W Z, L S, XJ L, C L, QD G, ZG Z: A Novel IncRNA HOXC-AS3 Acts as a miR-3922-5p Sponge to Promote Breast Cancer Metastasis. Cancer investigation 2020, 38(1):1-12.

24. Mason B, Flach S, Teixeira F, Manzano Garcia R, Rueda O, Abraham J, Caldas C, Edwards P, Laman H: Fbxl17 is rearranged in breast cancer and loss of its activity leads to increased global 0GIcNAcylation. Cellular and molecular life sciences: CMLS2020, 77(13):2605-2620.

25. Raducu M, Fung E, Serres S, Infante P, Barberis A, Fischer R, Bristow C, Thézénas M, Finta C, Christianson $\mathrm{J}$ et al: SCF (Fbxl17) ubiquitylation of Sufu regulates Hedgehog signaling and medulloblastoma development. The EMBO journal 2016, 35(13):1400-1416.

\section{Figures}


A

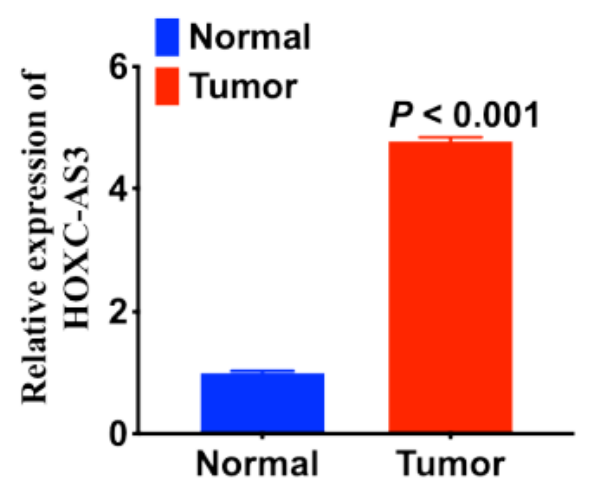

C
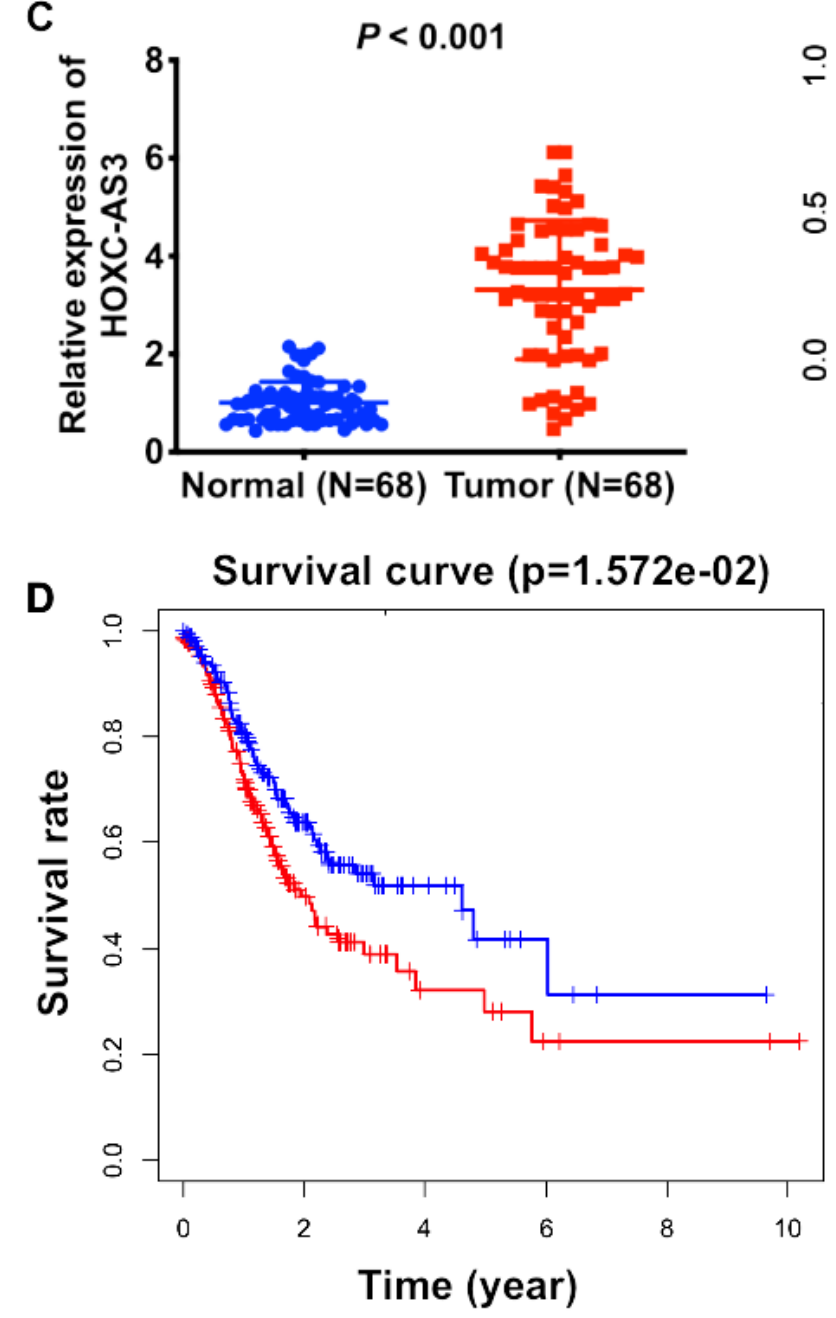

B

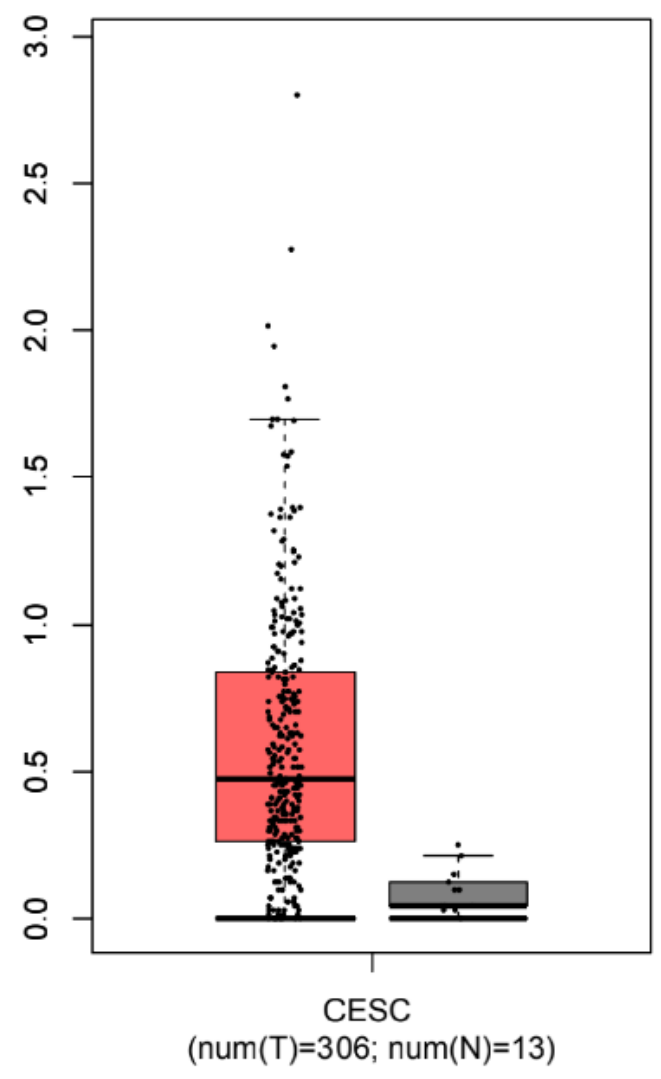

E

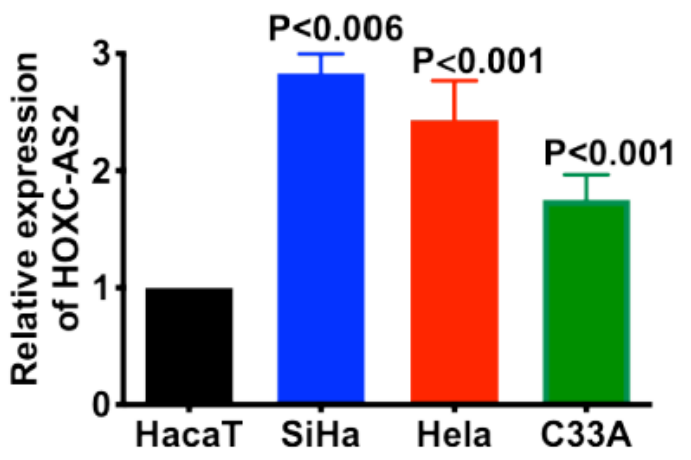

Figure 1

\section{Figure 1}

Upregulated expression of IncRNA HOXC-AS3 in cervical squamous cell carcinoma (CESC). (A) The expression of HOXC-AS3 in CESC tumor tissues and normal tissues was compared according to TCGA dataset. (B) GEPIA online tool showed the increased expression of HOXC-AS3 in CESC tumor tissues. (C) qRT-PCR was performed to detect the expression of HOXC-AS3 in 68 pairs of CESC tumor tissues and adjacent normal tissues. (D) Upregulated expression of HOXC-AS3 was correlated with poorer overall 
survival of CESC patients through TCGA database. (E) qRT-PCR results showed the relative expression of HOXC-AS3 in normal cell line (HacaT) and CESC cell lines (Siha, Hela and C33A).

A

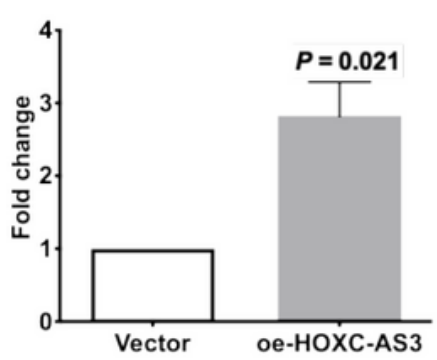

B

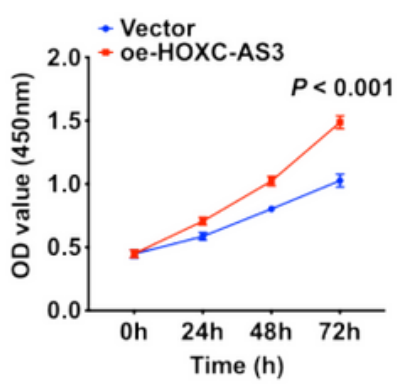

D

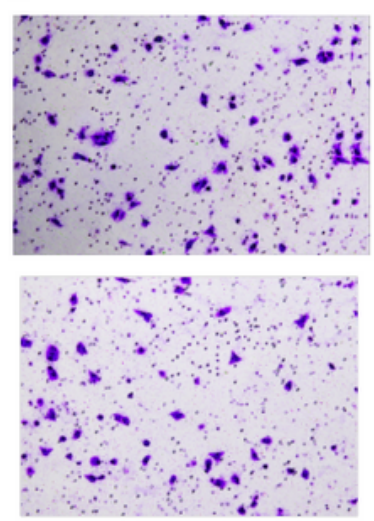

$\mathrm{F}$
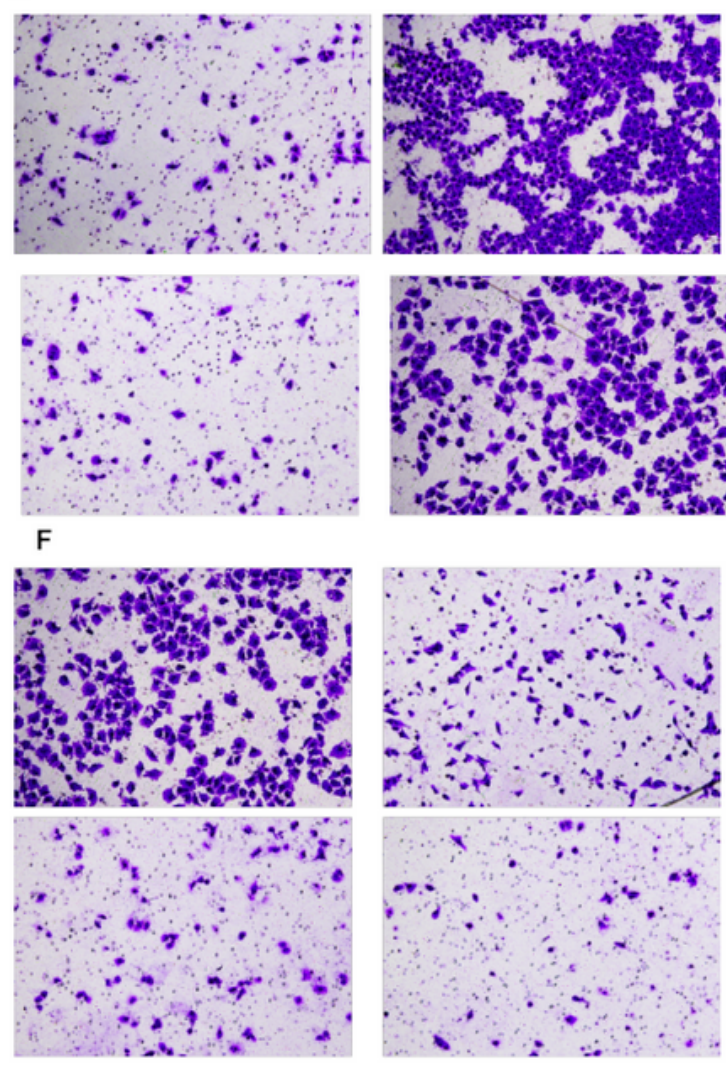

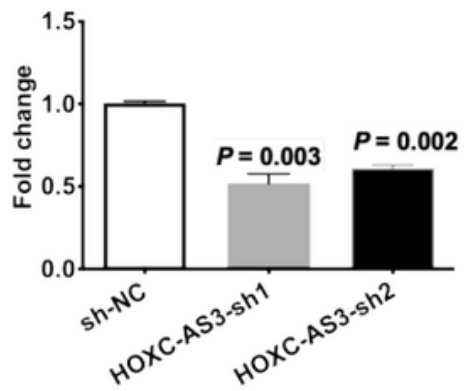

C $\quad$ shNC

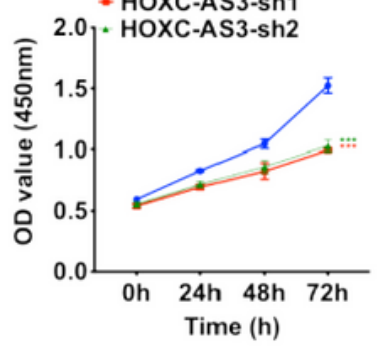

E

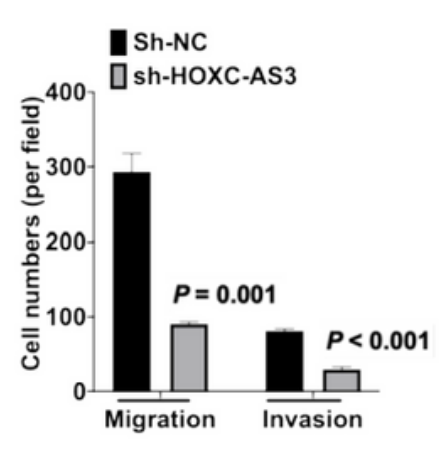

G

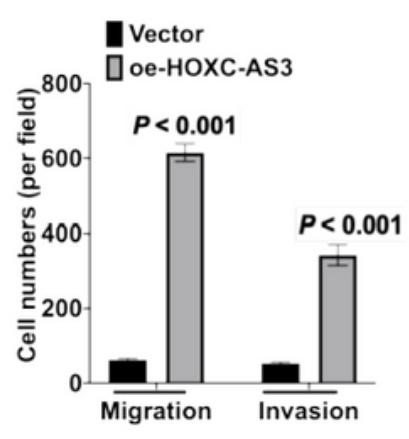

Figure 2

Figure 2

HOXC-AS3 promotes CESC cell proliferation, migration and invasion. (A) C33A cells was transfected with HOXC-AS3 overexpression plasmid. Upregulation of HOXC-AS3 expression in C33A cells. Siha cells were transfected with HOXC-AS3 shRNAs. Downregulation of HOXC-AS3 expression in Siha cells. Cell 
proliferation was assessed using CCK8 assay. (B) HOXC-AS3 overexpression promoted cell proliferation. (C) Silence of HOXC-AS3 inhibited the cell proliferation. Tumor cell migration and invasion were analyzed using transwell assays. (D and E) HOXC-AS3 overexpression significantly promoted the migration and invasion of cells. ( $F$ and $G$ ) Silence of HOXC-AS3 significantly inhibited the migration and invasion of CESC cells.

A

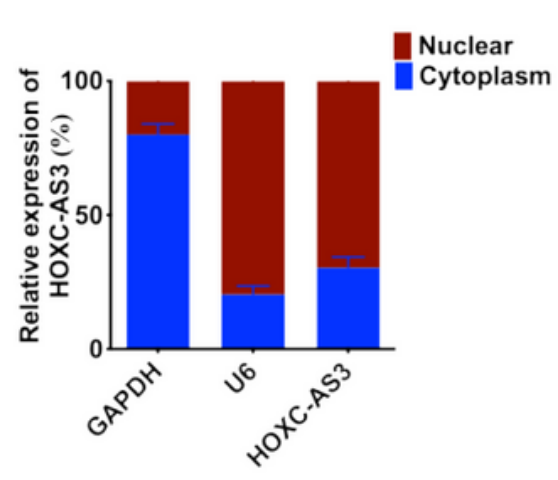

C

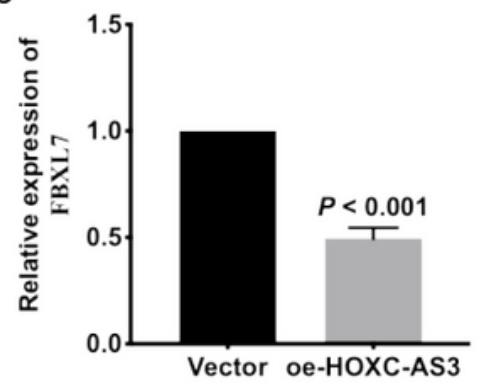

E

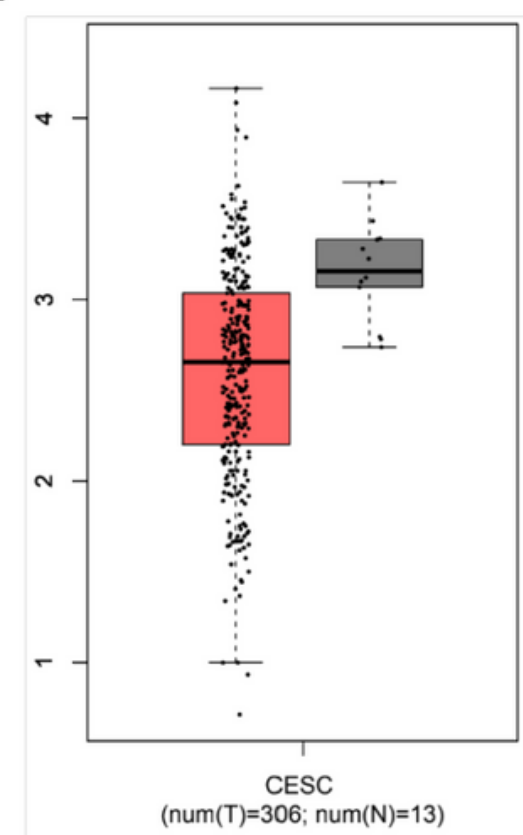

Figure 3

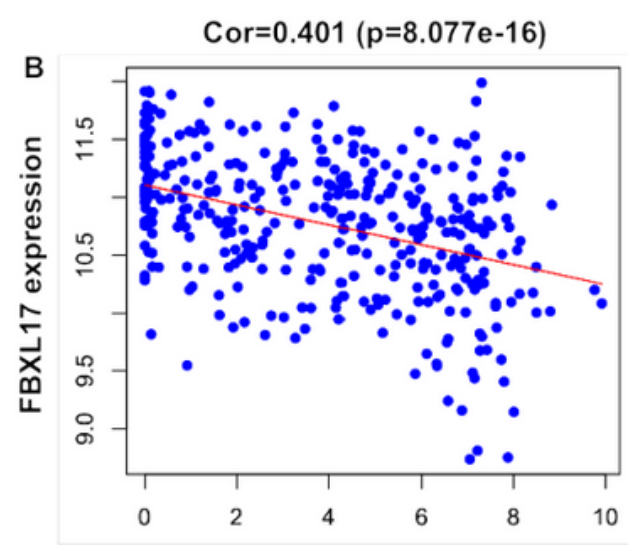

HOXC-AS3 expression

D

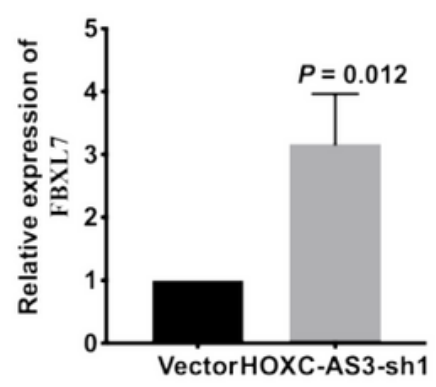

F

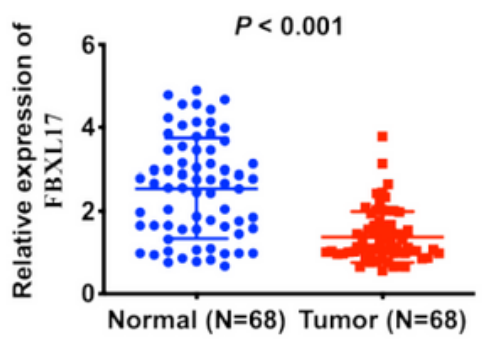

G

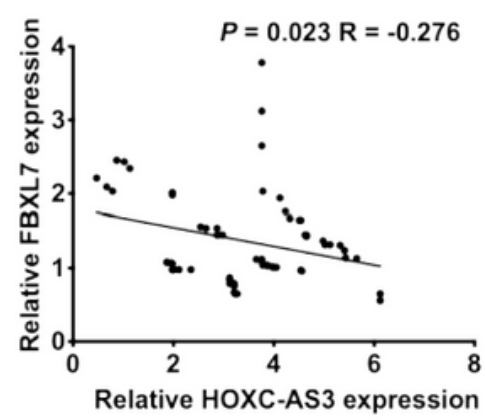

Figure 3 
The correlation between HOXC-AS3 and FBXL17 in CESC. (A) The subcelluar localization of HOXC-AS3 was detected using qRT-PCR. (B) There was positive correlation between HOXC-AS3 expression and FBXL17 expression. Data were extracted from TCGA database. (C) Overexpression of HOXC-AS3 upregulated the expression of FBXL17. (D) Silence of HOXC-AS3 significantly decreased the expression of FBXL17. (E) GEPIA online dataset showed the decreased expression of FBXL17 in CESC tumor tissues. (F) The expression of FBXL17 between 68 pairs of CESC tumor tissues and adjacent normal tissues were analyzed using qRT-PCR. (G) There was negative correlation between HOXC-AS3 expression and FBXL17 expression according to the qRT-PCR results.

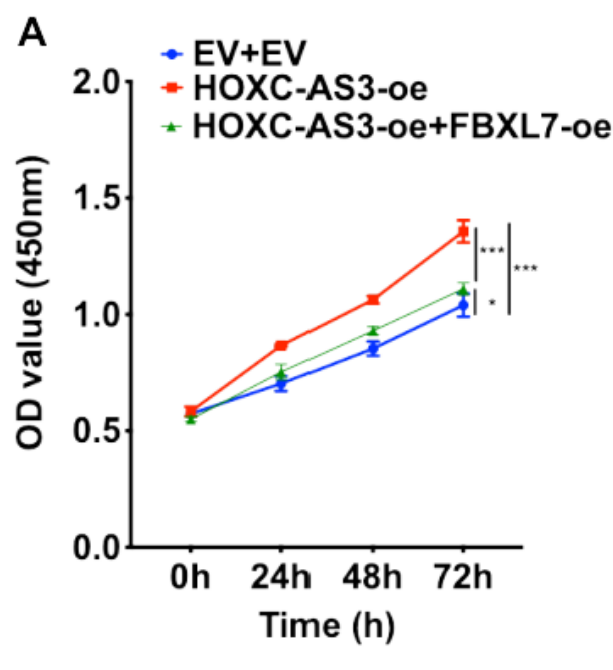

\section{C}

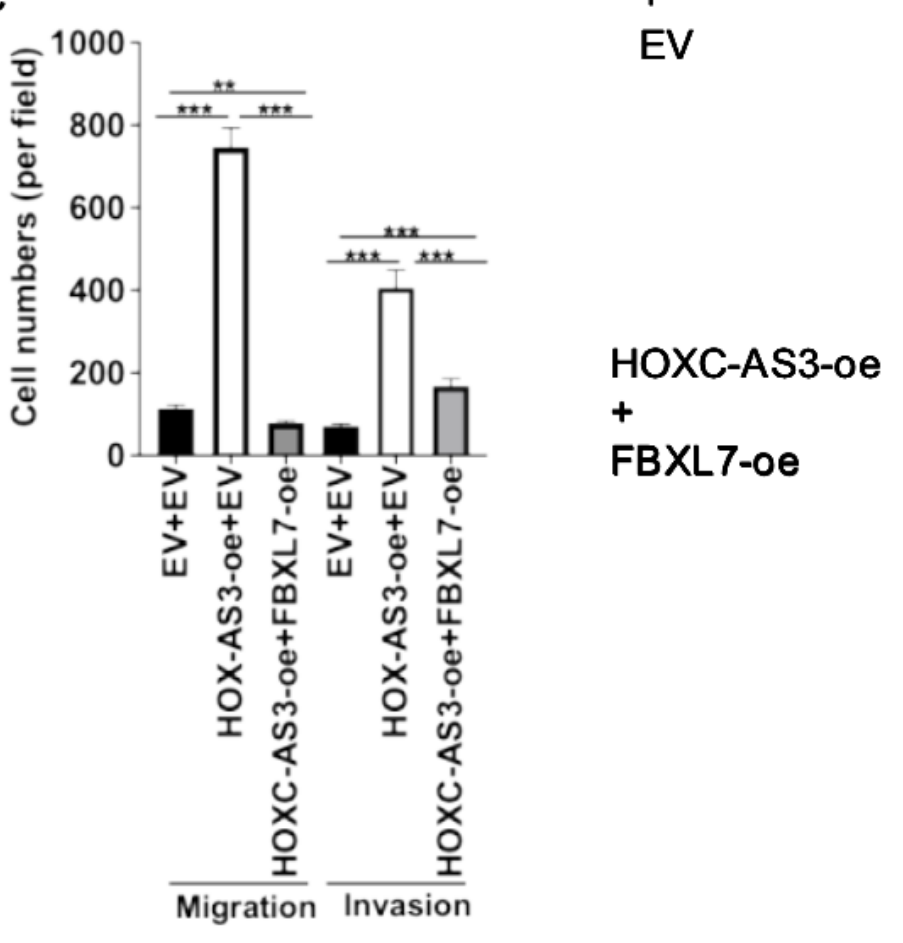

B

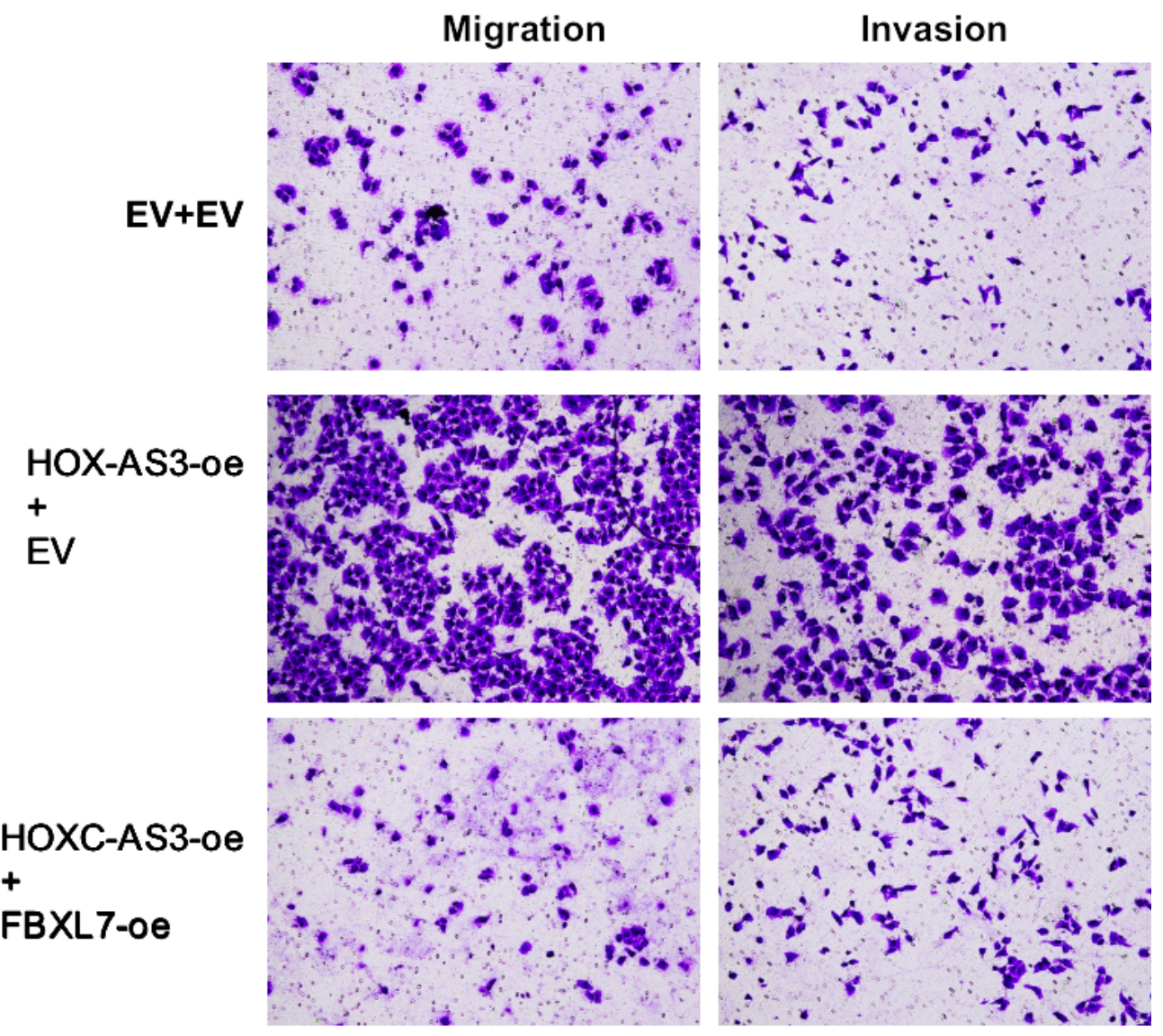

Figure 4

Figure 4 
HOXC-AS3 promotes cell migration and invasion through upregulation of FBXL17. (A) Concomitant overexpression of FBXL17 and HOXC-AS3 significantly inhibited the proliferation of CESC cells induced by HOXC-AS3 overexpression. (B and C) Overexpression of FBXL17 reversed the promotive effect of HOXC-AS3 overexpression on the migration and invasion of CESC cells.

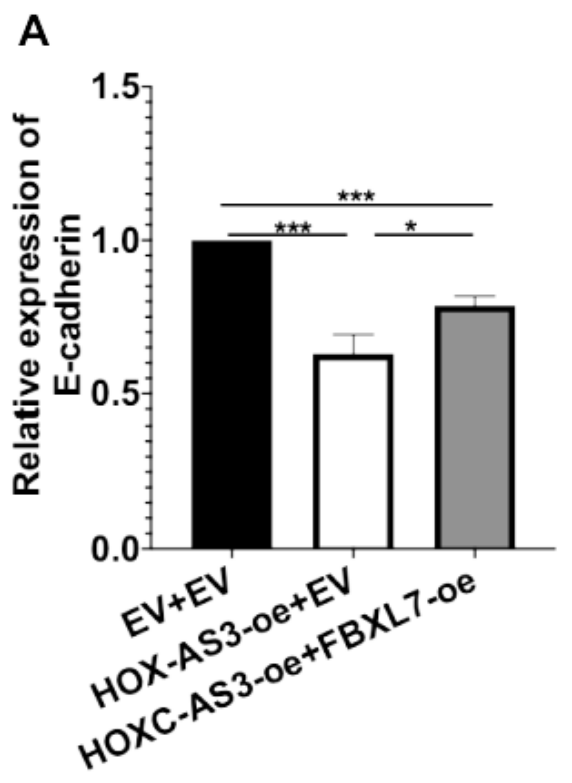

B

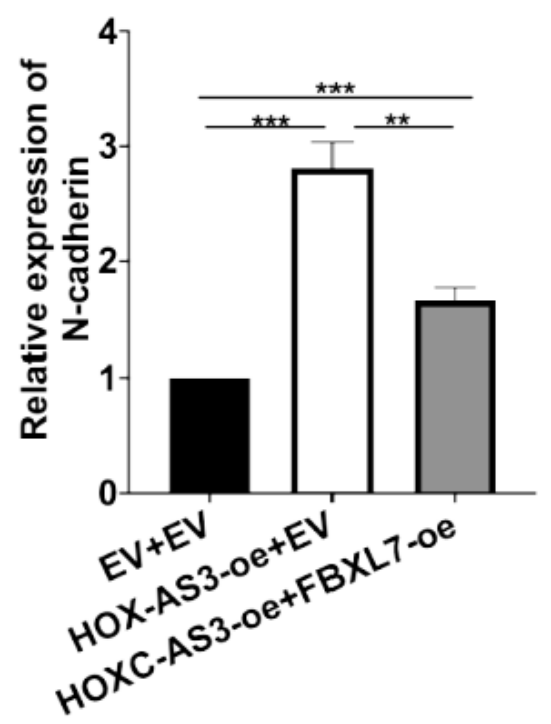

C

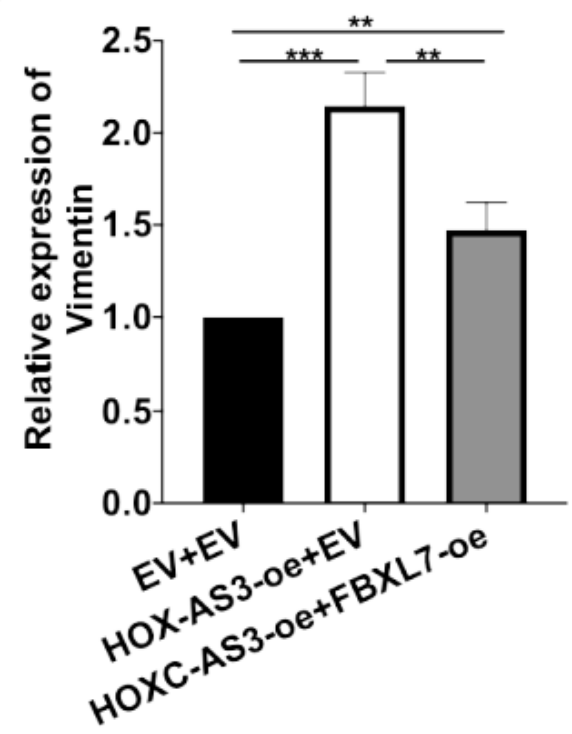

D

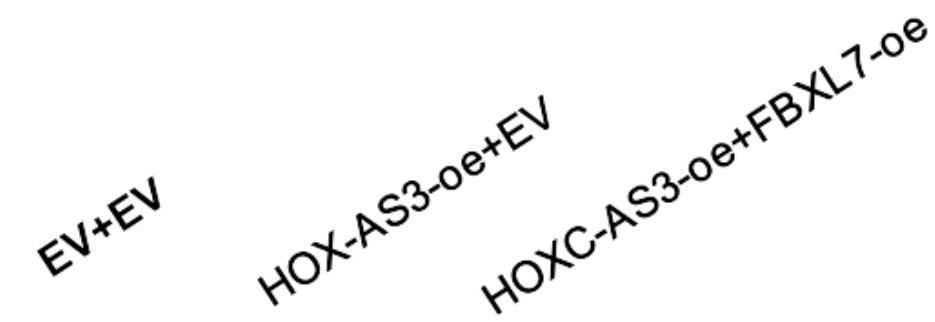

E-cadherin

$\mathrm{N}$-cadherin

Vimentin

$\beta$-actin

Figure 5

Figure 5

HOXC-AS3 interacted with FBXL17 to promote the EMT process. (A-C) qRT-PCR was performed to detect the expression of EMT related markers E-cadherin, N-cadherin and Vimentin. (D) Western blot analysis was performed to detect the protein expression levels of EMT related markers. 